Quim. Nova, Vol. 36, No. 9, 1416-1422, 2013

\title{
IMPLEMENTAÇÃO DE UM MÉTODO ROBUSTO PARA O CONTROLE FISCAL DE UMIDADE EM QUEIJO MINAS ARTESANAL. ABORDAGEM METROLÓGICA MULTIVARIADA
}

\author{
Bruno G. Botelho \\ Departamento de Química, ICEx, Universidade Federal de Minas Gerais, Av. Antônio Carlos, 6627, $31270-901$ Belo Horizonte \\ - MG, Brasil \\ Bruna A. P. Mendes \\ Instituto Mineiro de Agropecuária (IMA), BR 040, km 527, 32145-900 Contagem - MG, Brasil \\ Marcelo M. Sena*,\# \\ Departamento de Química, ICEx, Universidade Federal de Minas Gerais, Av. Antônio Carlos, 6627, 31270-901 Belo Horizonte \\ - MG, Brasil
}

Recebido em 7/2/13; aceito em 29/4/13; publicado na web em 1/7/13

\begin{abstract}
IMPLEMENTATION OF A ROBUST METHOD FOR QUALITY INSPECTION CONTROL OF ARTISANAL MINAS CHEESE. A MULTIVARIATE METROLOGICAL APPROACH. This study developed and validated a method for moisture determination in artisanal Minas cheese, using near-infrared spectroscopy and partial-least-squares. The model robustness was assured by broad sample diversity, real conditions of routine analysis, variable selection, outlier detection and analytical validation. The model was built from 28.5-55.5\% w/w, with a root-mean-square-error-of-prediction of 1.6\%. After its adoption, the method stability was confirmed over a period of two years through the development of a control chart. Besides this specific method, the present study sought to provide an example multivariate metrological methodology with potential for application in several areas, including new aspects, such as more stringent evaluation of the linearity of multivariate methods.
\end{abstract}

Keywords: quality control; NIRS; multivariate metrology.

\section{INTRODUÇÃO}

O Queijo Minas Artesanal (QMA) é um tradicional produto lácteo brasileiro, produzido em pequenas propriedades rurais por todo estado de Minas Gerais, que pode ser caracterizado como um queijo semi-duro, com alto teor de umidade, de coloração amarelo-pálida, formato cilíndrico e levemente ácido, características similares ao queijo português Serra da Estrela. Diferentemente do Queijo Minas Padrão, que é produzido de maneira industrial, com leite pasteurizado, o QMA deve ser produzido exclusivamente com leite cru, obtido da mesma propriedade de origem. ${ }^{1,2}$ Em função da variedade de procedimentos de fabricação desse queijo, uma grande diversidade é observada no produto final. Existem cinco regiões demarcadas como produtoras de QMA: Araxá, Campos das Vertentes, Cerrado, Serro e Serra da Canastra, ${ }^{3}$ porém, desde 2011, novas regiões podem ser reconhecidas. ${ }^{4}$ Atualmente, existem aproximadamente 30.000 produtores de QMA em cerca de 600 municípios por todo o estado de Minas Gerais. ${ }^{3}$

Até o presente momento, a ausência da legislação federal específica sobre o QMA restringe sua comercialização fora do estado de Minas Gerais. De acordo com a legislação estadual, o seu controle fiscal deve ser feito com relação ao teor de umidade (valor máximo permitido de $45,9 \%$ ), presença de amido, cuja adição é proibida, e teste da fosfatase, que atesta a não pasteurização do leite. ${ }^{5,6}$ Outros parâmetros físico-químicos, tais como teor de proteína ou gordura, não são exigidos. $\mathrm{O}$ método oficial sugerido pela legislação federal ${ }^{7}$ para a determinação de umidade em queijos é a dessecação em estufa a $105^{\circ} \mathrm{C}$, e as análises fiscais são realizadas em um laboratório oficial do estado, o Instituto Mineiro de Agropecuária (IMA). O método oficial é preciso, porém muito lento, podendo durar entre 3 e 6 horas por análise. ${ }^{8}$

*e-mail: marcsen@ufmg.br

\#Instituto Nacional de Ciência e Tecnologia em Bioanalítica (INCT Bio), 13083-970 Campinas - SP, Brasil
Apesar da ampla utilização dos métodos bromatológicos clássicos, eles apresentam algumas desvantagens consideráveis, como a utilização de grandes quantidades de solventes e reagentes, o alto custo relativo e o longo tempo de análise requerido para alguns procedimentos. Esses métodos não são apropriados para suprir a crescente demanda e a redução de custos desejável no controle de qualidade de produtos alimentícios. ${ }^{9}$ Uma estratégia que vem sendo aplicada com sucesso para superar esses problemas é a utilização conjunta de espectroscopia no infravermelho próximo (NIRS, do inglês near infrared spectroscopy) e calibração multivariada, possibilitando o desenvolvimento de métodos rápidos, de baixo custo, que não usam reagentes ou solventes, praticamente não necessitam de pré-processamento das amostras e não geram resíduos. Nos últimos anos, esta estratégia foi utilizada com sucesso na determinação de diversos parâmetros, como gordura, umidade e proteína, em alguns tipos de queijos e outros derivados de leite, como queijo Danbo, ${ }^{10}$ Cheddar, ${ }^{11}$ Emmental, ${ }^{12}$ Prato, ${ }^{13}$ Kefir, ${ }^{14}$ queijo fresco ${ }^{15}$ e processado. ${ }^{16}$ Entretanto, a grande maioria desses trabalhos analisou amostras de um número reduzido de produtores/origens/processos de fabricação, ou mesmo produziu as próprias amostras analisadas, o que não permitiu a incorporação de variância suficiente para o desenvolvimento de modelos adequados ao controle fiscal. Como já mencionado na literatura, ${ }^{17}$ muitos modelos multivariados propostos para a análise de alimentos não são suficientemente robustos para uso em análise de rotina, necessitando incorporar mais variância e ter sua confiabilidade garantida em condições reais. Nenhum dos artigos citados anteriormente desenvolveu um método multivariado que efetivamente fosse usado e testado no dia a dia de um laboratório de rotina, ou mesmo realizou um processo de validação analítica, garantindo sua confiabilidade.

O principal entrave para a validação de métodos multivariados tem sido a falta de guias específicos e regulamentação oficial apropriada. $\mathrm{Na}$ área de alimentos, tanto os guias oficiais brasileiros ${ }^{7}$ quanto os 
internacionais ${ }^{18-21}$ ignoram completamente aspectos multivariados intrínsecos ao desenvolvimento de métodos NIRS. Existe uma necessidade de harmonização de alguns conceitos, como seletividade, sensibilidade e curvas de calibração. Todos os guias tradicionais exigem seletividade total do sinal analítico, o que não faz sentido para calibração multivariada, a qual só tem utilidade na ausência de um sinal seletivo. Recomenda-se também a avaliação da linearidade através das curvas de calibração (resposta analítica versus concentração), que não são estritamente possíveis de obter em modelos multivariados. Dentre os esforços feitos nos últimos anos buscando a harmonização de aspectos univariados e multivariados, o conceito mais importante é o NAS (Net Analyte Signal, sinal analítico líquido), o qual possibilita separar uma parte específica do sinal total diretamente proporcional à propriedade de interesse, permitindo o cálculo de algumas figuras de mérito (FdM) multivariadas. A necessidade de harmonização entre a legislação vigente e a demanda de métodos multivariados tem sido discutida recentemente, de maneira geral ${ }^{22,23} \mathrm{e}$ em áreas específicas, como na análise farmacêutica ${ }^{24-26}$ e de produtos agrícolas. ${ }^{27}$

O objetivo deste artigo foi desenvolver e validar um método multivariado rápido e robusto para a determinação de umidade em QMA originados de mais de 80 diferentes produtores do estado de Minas Gerais. Este método se baseou em medidas de reflectância difusa por NIRS e no método dos mínimos quadrados parciais (PLS). Não foi possível o desenvolvimento de modelos para o controle fiscal dos outros dois parâmetros exigidos por lei para o QMA, uma vez que nenhuma amostra apresentou resultados acima do limite de quantificação para o amido ou teste positivo para a fosfatase. Mesmo assim, a metodologia desenvolvida se mostrou atraente, propiciando economia de tempo e recursos financeiros. A adequação do método desenvolvido para uso na rotina do IMA foi avaliada através de uma validação analítica multivariada completa, incluindo a estimativa de intervalos de confiança para as predições do modelo PLS, e a sua inspeção continuada, através do desenvolvimento de uma carta de controle. Mais do que esta aplicação específica, este trabalho visou apresentar uma metodologia metrológica multivariada completa para calibrações utilizando PLS, incluindo alguns aspectos inovadores, como uma avaliação mais rigorosa da linearidade deste tipo de método.

\section{VALIDAÇÃO ANALÍTICA DE MÉTODOS MULTIVARIADOS}

\section{Detecção de amostras anômalas (outliers)}

A detecção de amostras anômalas é extremamente importante no desenvolvimento de modelos multivariados, podendo ser essencial ao seu desempenho, especialmente em situações de calibração com amostras obtidas naturalmente, quando não se tem um controle completo de sua composição química (em oposição à situação de calibração planejada, quando as amostras podem ser preparadas de acordo com o desejado). A detecção de amostras anômalas pode ser feita utilizando diversos critérios, e neste trabalho, uma metodologia robusta $^{26,27}$ foi utilizada, avaliando valores de leverage extremos, resíduos elevados no bloco X (dados espectrais) e no bloco Y (valores de referência). Para a avaliação no bloco $\mathrm{X}$, amostras com $\mathrm{s}\left(\mathrm{e}_{\mathrm{i}}\right)>$ $2,0 \mathrm{~s}$ (e) foram removidas, onde s(e) é o desvio padrão total da informação espectral não modelada e $\mathrm{s}\left(\mathrm{e}_{\mathrm{i}}\right)$ é o desvio padrão especifico de cada amostra. Amostras anômalas no bloco Y são detectadas quando apresentam erro de predição duas vezes maior que o erro quadrático médio de calibração (RMSEC, Root Mean Square Error of Calibration). Por último, amostras com um leverage maior que o valor limite (três vezes o número de variáveis latentes (VL) mais um, dividido pelo número de amostras de calibração) também devem ser removidas. O leverage (h) é uma medida da influência de cada amostra no modelo e é calculado pela Equação 1, onde T é a matriz de escores das amostras de calibração, $\mathbf{t}_{\mathrm{i}}$ é o vetor de escores para a amostra i e A é o número de VL. Para todos os testes foi utilizado um nível de $95 \%$ de confiança. ${ }^{26,27}$

$$
\mathrm{h}_{\mathrm{i}}=\mathbf{t}_{\mathrm{A}, \mathrm{i}}^{\mathrm{T}}\left(\mathbf{T}_{\mathrm{A}}^{\mathrm{T}} \mathbf{T}\right)^{-1} \mathbf{t}_{\mathrm{A}, \mathrm{i}}
$$

\section{Sinal analítico líquido (NAS)}

O NAS é definido como a parte do sinal analítico exclusivamente relacionada com a propriedade de interesse e ortogonal ao espaço dos interferentes. Desenvolvido inicialmente para métodos de calibração direta, ${ }^{28}$ ele só foi utilizado efetivamente quando expandido para métodos de calibração inversa (PLS e PCR) ${ }^{29}$ e melhorado. ${ }^{30}$ Quando todos os interferentes podem ser modelados, o NAS pode ser estimado através de amostras do branco. ${ }^{29}$ Porém, neste trabalho isto não é possível, pois não é viável a produção de um queijo sem umidade. Nestes casos, o vetor NAS, $\hat{\mathbf{x}}_{A, i}^{\text {nas }}$, pode ser estimado para cada amostra i através do vetor de regressão de um modelo PLS com A variáveis latentes, b, de acordo com a Equação 2.

$$
\hat{\mathbf{x}}_{A, i}^{n a s}=\mathbf{b}\left(\mathbf{b}^{\mathrm{T}} \mathbf{b}\right)^{-1} \mathbf{b}^{\mathrm{T}} \mathbf{x}_{\mathrm{i}}
$$

A norma de cada vetor NAS fornece um escalar nâs, para cada amostra, que é análogo a um sinal analítico univariado. A estimativa do NAS é útil na validação multivariada, pois é utilizada no cálculo de algumas FdM, como seletividade (SEL), sensibilidade (SEN) e limites de detecção (LD) e quantificação (LQ). ${ }^{22,23}$

\section{Figuras de mérito (FdM)}

Um dos principais objetivos deste trabalho é propor a harmonização entre os aspectos pertinentes à calibração multivariada e os guias de validação utilizados em análises de alimentos, ${ }^{7,18-21}$ todos restritos a uma visão univariada. Para isto, as seguintes FdM foram estimadas: veracidade, precisão, linearidade, faixa de trabalho, SEL, SEN, sensibilidade analítica $(\gamma)$, viés e RPD (Residual Prediction Deviation, relação de desempenho do desvio). As estimativas de LD e LQ para este tipo de análise não são exigidas. A veracidade (termo atualizado, que substitui o antigo significado de exatidão, a qual é considerada atualmente o conjunto de veracidade mais precisão) ${ }^{20,31} \mathrm{de}$ métodos multivariados normalmente é avaliada através de parâmetros específicos não contemplados pelos guias tradicionais: o RMSEC e, principalmente, o RMSEP (Root Mean Square Error of Prediction), estimado com as amostras de validação externas. Além disso, é fundamental informar a faixa dos erros relativos de predição para as amostras individuais. Para se avaliar a precisão, não existe diferença entre os conceitos uni e multivariado. Ela é estimada como repetitividade, analisando-se seis replicatas de três amostras de diferentes concentrações no mesmo dia, e como precisão intermediária, com diferentes analistas analisando as mesmas amostras.

Considerando-se a impossibilidade de se obter uma curva de calibração para modelos multivariados, a linearidade é comumente avaliada através do coeficiente de correlação (r) do ajuste entre valores de referência e preditos. Porém, o valor de $r$ isoladamente não é capaz de assegurar a linearidade do modelo. ${ }^{21}$ É necessário também verificar a ausência de erros sistemáticos nos resíduos, o que quase sempre é feito subjetivamente pela inspeção visual de um gráfico. Com o objetivo de estabelecer uma avaliação mais objetiva da linearidade, este trabalho adaptou uma metodologia previamente descrita para métodos univariados utilizando mínimos quadrados ordinários, ${ }^{32}$ a qual garante a aleatoriedade dos resíduos através de 
testes estatísticos. O teste mais importante é o teste de Durbin-Watson (DW) ${ }^{33}$ no qual a ausência de autocorrelação é verificada através da comparação entre os desvios padrão (DP) dos resíduos ordenados e das diferenças sucessivas desses resíduos. Antes de se aplicar o DW, a normalidade e a homocedasticidade dos resíduos devem ser avaliadas, pelos testes de Ryan-Joiner (RJ) e Brown-Forsythe (BF), respectivamente. ${ }^{32}$

A faixa de trabalho foi definida como o intervalo no qual o método apresenta linearidade, veracidade e precisão aceitáveis. Algumas FdM multivariadas se baseiam no NAS. A SEL é a razão entre a norma do vetor NAS de cada amostra, ||nas $\|$, e a norma do respectivo espectro, $\left\|\mathbf{x}_{\mathrm{i}}\right\|$. Como esse valor varia para cada amostra, a média de todas elas é usada para representar o método. Entretanto, é importante salientar que a exigência de um sinal analítico seletivo, presente nos guias de validação oficiais, não se aplica a métodos quimiométricos. A SEN de métodos de calibração inversa é estimada como o inverso da norma do vetor dos coeficientes de regressão do modelo PLS (1/\|b||). Porém, a SEN é dependente da técnica analítica utilizada, e outra FdM, a $\gamma$, é mais adequada para a comparação com outros métodos. ${ }^{23} \mathrm{~A} \gamma$ é a razão entre a SEN e o ruído instrumental $(\varepsilon)$, o qual para métodos de reflectância difusa pode ser estimado como o desvio padrão combinado de espectros replicados do acessório vazio (uma placa de vidro fornecida pelo fabricante do equipamento). O inverso da $\gamma$ fornece uma estimativa da diferença mínima discernível pelo método, considerando-se o erro aleatório instrumental a única fonte de erros.

Baixos erros de predição somente não garantem a ausência de erro sistemático em um modelo. Para essa avaliação, a estimativa do viés é necessária, de acordo com a Equação $3 .^{34}$

$$
\text { Viés }=\sum_{i=1}^{n_{v}} \frac{\left(y_{i}{ }_{i}-\hat{y}_{i}\right)}{n_{v}}
$$

onde $\mathbf{y}^{\text {ref }}{ }_{\mathrm{i}}$ e $\hat{\mathbf{y}}_{\mathrm{i}}$ são os valores de referência e preditos, respectivamente, e $\mathrm{n}_{\mathrm{v}}$ é o numero de amostras de validação. Na sequência, o DP dos erros de validação é calculado e utilizado em um teste t para avaliar se o modelo apresenta viés estatisticamente significativo. Os valores de $t$ crítico e $t$ calculado são então comparados com o número apropriado de graus de liberdade. Uma FdM mais recente, utilizada para estimar a capacidade preditiva dos métodos, é a RPD, ${ }^{35}$ mais adequada para comparações em termos absolutos. A RPD é estimada para os conjuntos de calibração e validação de acordo com as Equações 4 e 5 , onde RMSECV é o erro quadrático médio de validação cruzada e $\mathrm{DP}_{\text {cal }}$ e $\mathrm{DP}_{\text {val }}$ são os DPs dos valores de referência dos conjuntos de calibração e validação, respectivamente.

$$
\begin{gathered}
\mathrm{RPD}_{\text {cal }}=\mathrm{DP}_{\text {cal }} / \mathrm{RMSECV} \\
\mathrm{RPD}_{\mathrm{val}}=\mathrm{DP}_{\mathrm{val}} / \mathrm{RMSEP}
\end{gathered}
$$

\section{Erros de predição específicos (EPE)}

A generalização da estimativa de incerteza dos valores preditos para modelos multivariados ainda é um assunto controverso. Existem diferentes propostas para a estimativa do EPE e as mais recentes são caracterizadas pela tendência de se afastarem do total rigor estatístico, priorizando a facilidade de cálculo e de utilização. ${ }^{36}$ Este trabalho utilizou a proposta errors in variables (EIV), ${ }^{36,37}$ que considera a estimativa de incerteza do método de referência e deve ser usada na ausência de viés. Esta proposta tem sido considerada menos sujeita a superestimar os efeitos do ruído nos dados ${ }^{36}$ do que outras propostas, como a sugerida pela ASTM..$^{34}$ A expressão EIV é mostrada na Equação 6, onde h é o leverage, definido anteriormente na Equação 1, e s $\left(y_{\text {ref }}\right)$ é a estimativa da incerteza do método de referência, estimada através de dados históricos.

$$
\mathrm{EPE}=\operatorname{RMSEC} \sqrt{(1+h)-\sigma\left(y_{\text {ref }}\right)^{2}}
$$

Uma vez que os fatores de um modelo PLS podem consumir mais de um grau de liberdade, o número de pseudo graus de liberdade $(\mathrm{PGL})^{38}$ é usado, juntamente com os EPE, na estimativa dos intervalos de confiança. O número de PGL é estimado através da Equação 7, onde $n_{c}$ é o número de amostras de calibração.

$$
\mathrm{PGL}=n_{c}\left(1-\frac{R M S E C}{R M S E C V}\right)
$$

\section{Carta de controle}

Uma vez desenvolvido, o método NIRS foi validado e aplicado na rotina do IMA para o controle de qualidade do QMA comercializado no estado de Minas Gerais. Desde então, para se avaliar o desempenho do modelo, uma carta de controle foi desenvolvida amostrando aleatoriamente uma amostra de QMA a cada quinzena (cerca de 5\% do total) e analisando-a por ambos os métodos, de referência e NIRS. A veracidade do método NIRS foi monitorada por essa carta de controle, cujos limites estão estabelecidos no guia de validação do MAPA. ${ }^{7}$ A normalidade, a homocedasticidade e a distribuição independente dos erros foram avaliadas pelos teste de RJ, BF e DW, respectivamente.

\section{PARTE EXPERIMENTAL}

\section{Instrumentação e processamento dos dados}

Todos os espectros foram obtidos utilizando um espectrofotômetro Perkin Elmer Spectrum 400 FTIR (Waltham, EUA), com acessório de reflectância difusa. Os programas MATLAB, versão R2011b (The MathWorks, Natick, EUA), e PLS Toolbox, versão 6.5 (Eigenvector Technologies, Manson, EUA), foram utilizados no processamento dos dados. Algumas rotinas próprias foram desenvolvidas para a detecção de amostras anômalas e cálculos de FdM.

\section{Materiais e amostras}

Areia purificada obtida de um fornecedor certificado foi usada no método de referência. Noventa e cinco amostras de QMA foram coletadas entre 2009 e 2010, originadas de diversos produtores de todo o estado de Minas Gerais. Os queijos foram transportados até o laboratório em temperatura menor que $4{ }^{\circ} \mathrm{C}$, deixados à temperatura ambiente por 30 minutos para atingirem equilíbrio térmico e imediatamente fatiados e analisados após a abertura da amostra. As fatias foram obtidas de partes internas da peça de queijo, menos susceptíveis à degradação.

\section{Procedimentos}

Os valores de referência do teor de umidade foram obtidos pelo método de dessecação em estufa, ${ }^{39}$ sem replicatas. Três gramas de cada amostra foram pesadas e misturadas homogeneamente com a mesma quantidade de areia purificada previamente seca, para prevenir a formação de crostas e acelerar o processo de secagem. As amostras foram secas a $105^{\circ} \mathrm{C}$ até atingir peso constante. Uma fatia de cada amostra foi colocada em uma placa de petri e seu espectro registrado entre 1000 e $2500 \mathrm{~nm}$, com passo de $2 \mathrm{~nm}$ e resolução de $8 \mathrm{~cm}^{-1}$, em 32 varreduras. Para se obter uma estimativa do ruído instrumental, 10 espectros de uma placa de petri vazia foram obtidos nas mesmas condições. Todas as análises foram realizadas em um ambiente com temperatura controlada $\left(22 \pm 1^{\circ} \mathrm{C}\right)$. Como mencionado anteriormente, após a validação analítica, o método desenvolvido foi implementado 
na rotina do controle de qualidade do IMA. Durante dois anos, 56 amostras foram determinadas por ambos os métodos (referência e NIRS), para se monitorar o desempenho do modelo pós-validação.

\section{RESULTADOS E DISCUSSÃO}

\section{Desenvolvimento e otimização do modelo PLS}

A Figura 1 mostra os espectros das 95 amostras de QMA. É possível notar que as bandas mais largas e intensas ocorrem próximas a 1.450 e $1.900 \mathrm{~nm}$, que correspondem ao primeiro sobretom de estiramento de $\mathrm{O}-\mathrm{H}$ e à banda de combinação de estiramento e deformação angular de $\mathrm{O}-\mathrm{H}$ de água, respectivamente. ${ }^{40}$ Dois terços das amostras foram separadas no conjunto de calibração (63) e o terço restante foi usado no conjunto de validação (32), utilizando o algoritmo de Kennard-Stone (KS). Esse algoritmo garante que as amostras mais representativas estejam no conjunto de calibração, através de uma varredura uniforme dos dados espectrais. ${ }^{41}$ Os espectros foram pré-processados com correção de espalhamento multiplicativo (MSC), para a remoção de desvios de linha base não linear causados pelo espalhamento multiplicativo da luz, fenômeno comum em medidas de reflectância difusa, ${ }^{42}$ e então centrados na média. O número de VL de cada modelo foi selecionado por validação cruzada em blocos contínuos (7 divisões). O melhor modelo inicialmente foi sugerido com $5 \mathrm{VL}$, considerando toda a região espectral. Este número de VL pode parecer alto, mas reflete a grande variabilidade contida nas amostras de queijo, obtidos de uma grande variedade de produtores. No entanto, a restrição da região espectral irá diminuir o número de VL, como será visto a seguir. Através dos coeficientes de regressão do modelo (Figura 2), é possível observar que as regiões espectrais mais importantes para o modelo estão relacionadas com vibrações de $\mathrm{O}-\mathrm{H}$, com valores mais positivos próximos a 1450 e $1900 \mathrm{~nm}$. Também é interessante ressaltar dois picos negativos próximos a 1200 e 1730 $\mathrm{nm}$, que podem ser atribuídos ao segundo e ao primeiro sobretons das

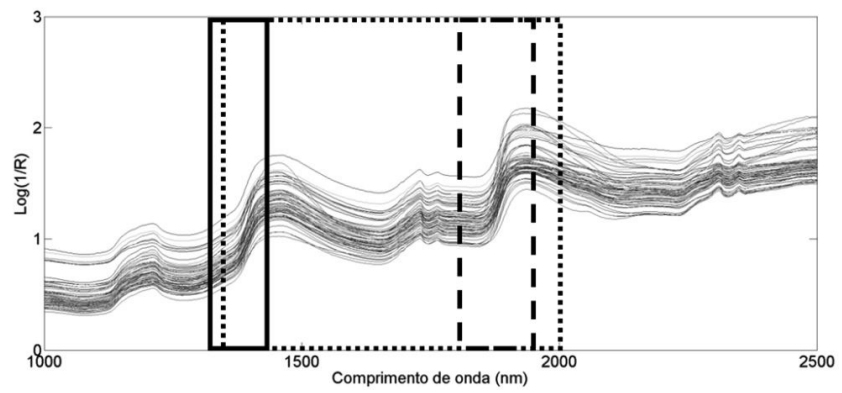

Figura 1. Espectros NIR das 95 amostras de queijo Minas artesanal. As regiões espectrais utilizadas nos modelos locais estão em destaque: região 1 (linha pontilhada), região 2 (linha sólida) e região 3 (linha tracejada)

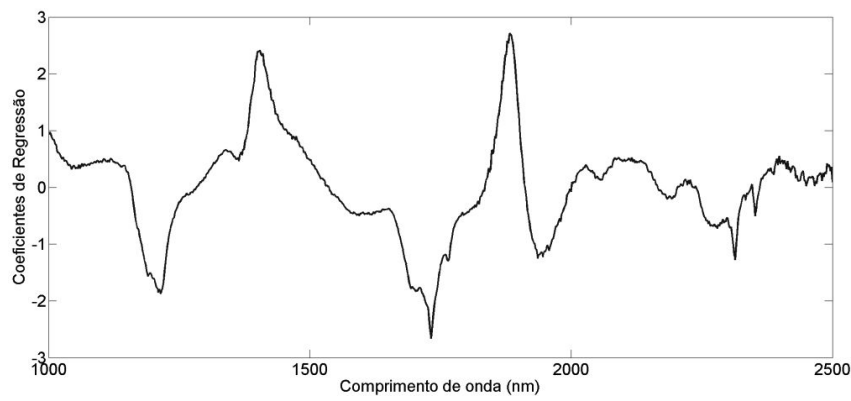

Figura 2. Coeficientes de regressão para o modelo de determinação de umidade usando os espectros inteiros ligações $\mathrm{C}-\mathrm{H}$, respectivamente ${ }^{40}$ e estão associadas a carboidratos, proteínas e gorduras, constituintes inversamente correlacionados com o teor de umidade desses queijos.

O modelo foi otimizado simultaneamente pela detecção de outliers e seleção de variáveis através da construção de modelos locais restritos às regiões espectrais de maior relevância preditiva, observadas na Figura 2. Três diferentes regiões foram avaliadas (Figura 1): região 1, entre 1400 e $2000 \mathrm{~nm}$, incluindo ambas as bandas relacionadas a ligações O-H; região 2, entre 1360 e 1440 nm; e região 3, entre 1800 e $1940 \mathrm{~nm}$. A seleção do melhor modelo foi feita buscando-se menores erros de predição e maiores valores de RPD (Tabela 1). O modelo selecionado foi obtido com 3 VL para a região 3, que está relacionada às bandas de combinação de O-H. Os quatro modelos gerados foram submetidos à detecção de outliers e os resultados são mostrados na Tabela 2 . O conjunto de validação só foi otimizado após a detecção de outliers na calibração. De acordo com os guias de validação brasileiros ${ }^{7}$ e internacionais, ${ }^{43}$ outliers podem ser removidos até um máximo de $22,2 \%$ (2/9) do número total de amostras. Treze amostras foram retiradas do conjunto de calibração (20,6\%): três por alto leverage, uma por alto resíduo espectral e nove por alto resíduo em Y. No conjunto de validação, sete amostras foram removidas $(21,8 \%)$, todas por altos resíduos em Y. O fato da maioria dos outliers ter sido detectada com base nos altos resíduos de Y é um indicativo da possível presença de erros nas medidas de referência, uma vez que estas são obtidas sem replicatas. Os outliers identificados com altos valores de leverage indicam amostras de composição muito diferente, um problema relacionado às suas origens (locais/ produtores). É importante ressaltar a necessidade de uma detecção de outliers tão rígida, pois o método desenvolvido tem como objetivo atender às exigências preconizadas pelos guias de validação oficiais. O modelo PLS final foi construído com 50 amostras de calibração e 25 de validação, utilizando 3 VL, e explicando 98,3\% da variância em X e 95,3\% em Y.

Tabela 1. Seleção de variáveis. Comparação entre modelos locais e o modelo utilizando o espectro inteiro

\begin{tabular}{lcccc}
\hline & $\begin{array}{c}\text { Espectro } \\
\text { inteiro }\end{array}$ & Região 1 & Região 2 & Região 3 \\
\hline RMSEC (\%) & 1,4 & 1,7 & 2,3 & 1,3 \\
$\operatorname{RMSECV~(\% )~}$ & 2,0 & 2,0 & 2,5 & 1,6 \\
$\operatorname{RMSEP}(\%)$ & 2,0 & 1,9 & 2,3 & 1,6 \\
$\operatorname{RDP}_{\text {cal }}$ & 3,4 & 3,5 & 2,9 & 4,5 \\
$\mathrm{RDP}_{\text {val }}$ & 2,1 & 2,1 & 1,9 & 2,4 \\
\hline
\end{tabular}

Tabela 2. Otimização do modelo local selecionado (região 3) através da detecção de amostras anômalas (resultados finais em itálico)

\begin{tabular}{lcccc}
\hline Modelo & $1^{\circ}$ & $2^{\circ}$ & $3^{\circ}$ & $4^{\circ}$ \\
\hline Amostras de calibração & 63 & 58 & 54 & 50 \\
Amostras de validação & 32 & 32 & 32 & 25 \\
Número de VL & 2 & 2 & 2 & 3 \\
RMSEC (\%) & 3,1 & 2,8 & 1,9 & 1,3 \\
RMSEP $(\%)$ & 4,6 & 4,8 & 5,0 & 1,6 \\
\hline
\end{tabular}

\section{Validação analítica multivariada}

A Tabela 3 apresenta as FdM calculadas para o modelo otimizado. A seguir, cada uma será discutida individualmente.

A linearidade foi estimada através do ajuste da curva dos valores de referência contra os valores preditos, cujos resíduos são 
Table 3. Figuras de mérito estimadas para o método de determinação de umidade em QMA

\begin{tabular}{|c|c|c|}
\hline Figuras de Mérito & Parâmetros & Valores \\
\hline Veracidade & Erros relativos (máx/min) & $-7,4 / 8,1 \%$ \\
\hline \multirow[t]{6}{*}{ Precisão } & DPR repetitividade ${ }^{\mathrm{a}}$ & $2,7 \%$ \\
\hline & & $2,0 \%$ \\
\hline & & $2,3 \%$ \\
\hline & DPR precisão intermediária ${ }^{a}$ & $3,5 \%$ \\
\hline & & $3,0 \%$ \\
\hline & & $3,8 \%$ \\
\hline \multirow[t]{4}{*}{ Linearidade } & Teste de Durbin-Watson & 1,84 \\
\hline & Inclinação ${ }^{\mathrm{b}}$ & $1,00 \pm 0,06$ \\
\hline & Intercepto $^{\mathrm{b}}$ & $0,00 \pm 2,69$ \\
\hline & Coeficiente de correlação ${ }^{b}$ & 0,9766 \\
\hline Faixa de trabalho & & $28,5-55,5 \%$ \\
\hline Seletividade & & 0,28 \\
\hline Sensibilidade & & $0,016^{\mathrm{c}}$ \\
\hline Sensibilidade Analítica $(\gamma)$ & & $3,2 \%^{-1}$ \\
\hline$\gamma^{1}$ & & $0,3 \%$ \\
\hline Viés & & $0,641 \pm 1,625 \%$ \\
\hline
\end{tabular}

${ }^{\mathrm{a}}$ Resultado de três amostras em 3 diferentes níveis de concentração. ${ }^{\mathrm{b}}$ Parâmetros da curva ajustada às amostras de calibração. ${ }^{c}$ Valores expressos como a relação entre $\log (1 / \mathrm{R})$ e \%.

mostrados na Figura 3. Para confirmar a distribuição aleatória desses resíduos, testes estatísticos apropriados foram feitos, verificando sua normalidade (RJ), homocedasticidade (BF) e ausência de correlação (DW), todos com 95\% de confiança. Todos os resultados indicaram a aleatoriedade dos resíduos. Em particular, o valor de DW calculado, 1,84 , está dentro dos limites de aceitação $(1,50-2,50)$, garantindo a independência dos resíduos. Uma vez considerado válido o ajuste linear, os parâmetros da curva (Tabela 3) podem ser usados para expressar a linearidade do método.

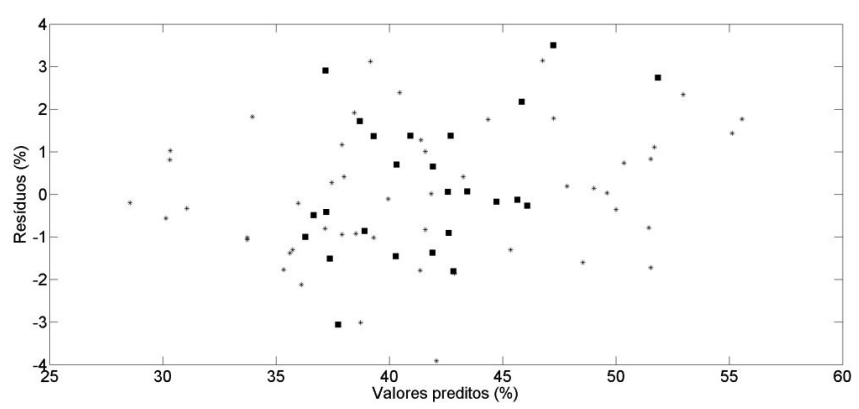

Figura 3. Resíduos das amostras de calibração (asteriscos) e de validação (quadrados)

A veracidade do método foi avaliada através dos parâmetros referentes aos erros absolutos (Tabela 1), principalmente o RMSEP de $1,6 \% \mathrm{~m} / \mathrm{m}$. Também foram avaliados os erros relativos de predição, entre $-7,4$ e $8.1 \%$, valores que estão de acordo com os limites preconizados pelo guia de validação do MAPA, entre -20 e $+10 \%$. $^{7}$ $\mathrm{O}$ método foi ainda considerado preciso, com Desvio Padrão Relativo (DPR) entre 2,0 e 2,7\% para a repetitividade e entre 3,0 e $3,8 \%$ para a precisão intermediária. Estes valores estão de acordo com a legislação, ${ }^{7}$ que estabelece limites máximos de $4 \%$ para a repetitividade e $10 \%$ para a precisão intermediária, considerando o nível de concentração da propriedade de interesse (acima de $100 \mathrm{~g}$ $\mathrm{kg}^{-1}$ ). Os resultados de precisão e veracidade garantiram a exatidão do método. ${ }^{20,31}$ Considerando as avaliações de linearidade e exatidão, a faixa de trabalho foi estabelecida entre 28,5 e $55,5 \% \mathrm{~m} / \mathrm{m}$ de umidade.

Como mencionado na sessão teórica, a SEL de métodos multivariados não possui significado prático em termos de validação analítica. Para este método, seu valor médio foi de $28 \%$, o que indica a fração do sinal analítico utilizada na predição da umidade. A SEN não é um parâmetro adequado para a comparação entre métodos analíticos. Por isso, um parâmetro mais útil, a $\gamma$, foi calculado (Tabela $3)$, considerando um $\varepsilon$ de 0,005 . O inverso da $\gamma(0,3 \%)$ fornece a mínima concentração discernível pelo método, considerando o erro instrumental aleatório como única fonte de erros.

Considerando a estimativa do viés mostrada na Tabela 3 , um valor de t de 1,96 foi obtido, abaixo do t crítico $(2,06)$, calculado com 25 graus de liberdade (número de amostras de validação) no nível de $95 \%$ de confiança. O método pôde então ser considerado livre de tendências sistemáticas. De acordo com a literatura, ${ }^{35}$ bons modelos de calibração devem possuir um valor de RPD acima de 2,4, enquanto valores entre 2,4 e 1,5 são considerados satisfatórios. Modelos com RPD menor que 1,5 não devem ser utilizados. Observando os resultados da Tabela 1 (região 3), pode-se afirmar que o método apresentou bons RPD tanto para a calibração quanto para a validação.

\section{EPE}

O EPE estimado pela equação EIV (Equação 6) para as amostras de validação variou entre 2,9 e 3,2\%. A Figura 4 mostra os valores de referência e os valores preditos, com os respectivos intervalos de confiança, para cada amostra de validação. Neste cálculo, a incerteza do método de referência, $0,1 \%$, foi obtida de dados históricos. De acordo com a Equação 7, 9 pseudo graus de liberdade foram usados no cálculo dos intervalos de confiança $(\mathrm{t}=2,03)$. Apenas duas das 25 amostras não estão contidas nestes intervalos, representando uma concordância de $92 \%$ nos resultados das predições.

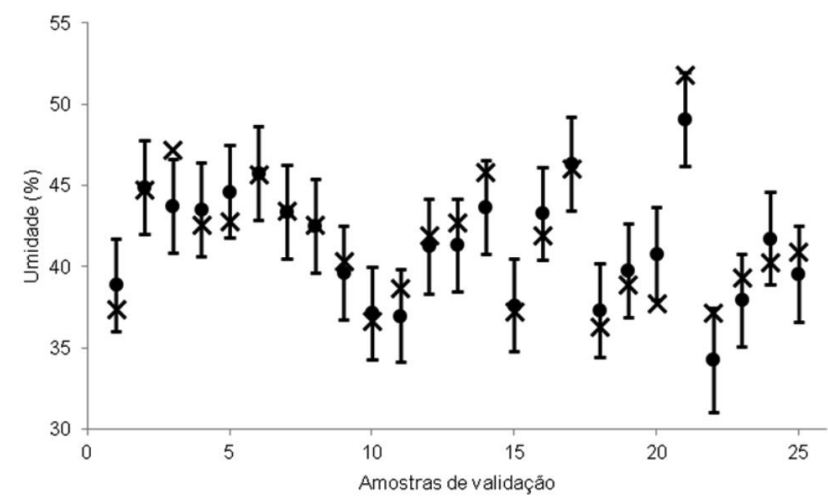

Figura 4. Intervalos de confiança para as amostras preditas pelo modelo PLS, estimados com base no EPE das amostras de validação. Valores preditos (círculos) e de referência (letras $x$ )

\section{Carta de controle para monitorar a estabilidade do modelo}

Após a validação analítica, o método foi utilizado no controle de qualidade fiscal de rotina do QMA. Uma vez que a estabilidade do modelo deve ser constantemente monitorada, uma carta de controle foi desenvolvida com os erros relativos das predições de 56 amostras, analisadas durante um período de dois anos após a sua implementação. Esta carta é mostrada na Figura 5. Os limites indicados foram baseados na legislação brasileira, ${ }^{7}$ que estabelece que dois terços das 
amostras devem apresentar erros menores que os limites de alerta $( \pm 15 \%)$, enquanto nenhuma amostra deve apresentar erros acima dos limites de ação $( \pm 33 \%)$. Como pode ser observado, apenas três amostras $(5,4 \%)$ estão entre os limites de alerta e de atenção. Os erros relativos também foram considerados normais e homocedásticos no nível de $95 \%$ de confiança, de acordo com os testes RJ e BF, respectivamente. A independência dos erros foi confirmada pelo teste DW, com valor de 2,03. Estes resultados indicam que o modelo se manteve satisfatório durante o período estudado. Caso a carta de controle venha a indicar no futuro a perda de estabilidade do modelo, será necessário o uso de métodos de atualização, como a regularização de Tikhonov. ${ }^{44}$

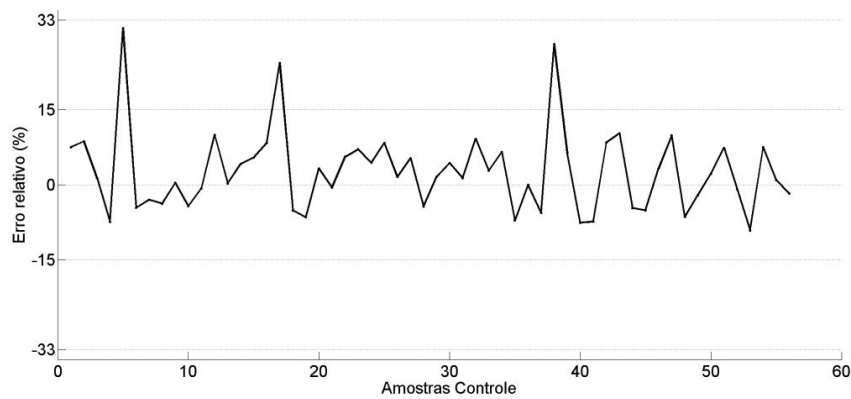

Figura 5. Carta de controle desenvolvida para estimar a estabilidade do modelo ao longo do tempo

\section{CONCLUSÃO}

Um método robusto baseado em medidas de reflectância difusa no NIR foi desenvolvido para a determinação de umidade em queijo Minas artesanal, sob condições reais de um laboratório de controle de qualidade fiscal. Ele é mais simples e aproximadamente 80 vezes mais rápido que o método de referência, reduzindo o gasto com mão de obra, que é a parcela mais onerosa do custo final da análise, além de não necessitar de reagentes, como a areia purificada, produto cujo preço excede $\mathrm{R} \$ 1000$ por quilo (como são consumidos $3 \mathrm{~g}$ por medida, estima-se que só com a economia deste reagente deixa-se de gastar cerca de R \$3,10 em cada análise). Este método foi validado de acordo com guias brasileiros e internacionais, sendo considerado linear, exato, suficientemente sensível e livre de viés. Após a implementação do modelo, uma carta de controle foi desenvolvida e utilizada para confirmar sua estabilidade por um período de dois anos. Outro objetivo, além desta aplicação específica, foi demonstrar o desenvolvimento de uma metodologia de metrologia multivariada de potencial interesse na análise de alimentos e em outras áreas. Esta metodologia empregou uma detecção robusta de amostras anômalas, seleção de variáveis espectrais, estimativa de incerteza para as amostras de validação e harmonização entre os conceitos univariados e multivariados de validação analítica, incluindo uma avaliação mais rigorosa da linearidade de modelos multivariados através de testes estatísticos como o de Durbin-Watson.

\section{AGRADECIMENTOS}

B.G. Botelho agradece à CAPES e ao CNPq pela bolsa de Doutorado.

\section{REFERÊNCIAS}

1. Furtado, M. M. A.; A Arte e a Ciência do Queijo, 2a ed., Ed. Globo S.A.: São Paulo, 1991.

2. Nogueira, M. C. L.; Lubachevsky, G.; Rankin, S. A.; LWT Food Sci. Technol. 2005, 38, 555.
3. http://www.agricultura.mg.gov.br/noticias/1812-lei-reconhecenovas-regioes-produtoras-de-queijo-minas-artesanal, acessada em Junho 2013.

4. Governo do Estado de Minas Gerais. Lei No 19.492, Brasil, 2011.

5. Governo do Estado de Minas Gerais. Regulamento da Lei $N^{o} 14185$, Decreto $N^{\circ}$ 42645, Brasil, 2002.

6. Governo do Estado de Minas Gerais. Decreto $N^{o} 44864$, Brasil, 2008.

7. Ministério da Agricultura, Pecuária e Abastecimento (MAPA). Secretaria de Defesa Agropecuária. Instrução Normativa No 24, Brasil, 2009.

8. Bradley, R. L.; Vanderwarn, M. A.; J. AOAC Int. 2001, 84, 570.

9. Rodriguez-Otero, J. L.; Hermida, M.; Centeno, J.; J. Agric. Food Chem. 1997, 45, 2815.

10. Wittrup, C.; Nørgaard, L.; J. Dairy Sci. 1998, 81, 1803.

11. Blazquez, C.; Downey, G.; O’Donnell, C.; O'Callaghan, D.; Howard, V.; J. Near Infrared Spectrosc. 2004, 12, 179.

12. Karoui, R.; Mouazen, A.M.; Dufour, E.; Pillonel, L.; Schaller, E.; Picque, D.; De Baerdemaeker, J.; Bosset, J. O.; Eur. Food Res. Technol. 2006, 223, 44.

13. Sauer-Leal, E.; Okada, F. M.; Peralta-Zamora, P.; Quím. Nova 2008, 31, 1621.

14. Ntsame Affane, A. L.; Fox, G. P.; Sigge, G. O.; Manley, M.; Britz, T. J.; Int. Dairy J. 2011, 21, 896.

15. Lucas, A.; Andueza, D.; Rock, E.; Martin, B.; J. Agric. Food Chem. 2008, 56, 6801

16. Pi, F.; Shinzawa, H.; Ozaki, Y.; Han, D.; Int. Dairy J. 2009, 19, 624.

17. Huang, H.; Yu, H.; Xu, H.; Ying, Y.; J. Food Eng. 2008, 87, 303.

18. International Conference on Harmonization (ICH). Tripartite Guideline - Q2B Validation of Analytical Procedures: Methodology, Fed. Regist., 62 FR 27464, EUA, 1997.

19. Food and Agriculture Organization (FAO). Validation of analytical methods for food control. Report of a Joint FAO/IAEA Experte Consultation, FAO Food and Nutrition Paper $N^{\circ}$ 68, Roma, Itália, 1998.

20. European Comission (EC). Directive 657. Comission Decision of 12 August 2002 implementing Council Directive 96/23/EC concerning the performance of analytical methods and the interpretation of results, Official Journal, L221, 8-36, Bruxelas, Bélgica, 2002.

21. Thompson, M.; Ellison, S. L. R.; Wood, R.; Pure Appl. Chem. 2002, 74, 835.

22. Olivieri, A. C.; Faber, N. M.; Ferré, J.; Boqué, R.; Kalivas, J. H.; Mark, H.; Pure Appl. Chem. 2006, 78, 633.

23. Valderrama, P.; Braga, J. W. B.; Poppi, R. J.; Quim. Nova 2009, 32, 1278.

24. Braga, J. W. B.; Poppi, R. J.; Quím. Nova 2004, 27, 1004.

25. Silva, M. A. M.; Ferreira, M. H.; Braga, J. W. B.; Sena, M. M.; Talanta 2012, 89, 342 .

26. Ferreira, M. H.; Braga, J. W. B.; Sena, M. M.; Microchem. J. 2013, 109, 158.

27. Valderrama, P.; Braga, J. W. B.; Poppi, R. J.; J. Agric. Food Chem. 2007, 55,8331 .

28. Lorber, A.; Anal. Chem. 1986, 58, 1167.

29. Lorber, A.; Faber, K.; Kowalski, B. R.; Anal. Chem. 1997, 69, 1620.

30. Bro, R.; Andersen, C. M.; J. Chemom. 2003, 17, 646.

31. INMETRO, Vocabulário Internacional de Metrologia - Conceitos fundamentais e gerais e termos associados (VIM 2012), $1^{\text {a }}$ ed. luso-brasileira, Portaria n. ${ }^{\circ}$ 232, Brasília, Brasil, 2012.

32. Souza, S. V. C.; Junqueira, R. G.; Anal. Chim. Acta 2005, 552, 25.

33. Durbin, J.; Watson, G. S.; Biometrika 1951, 38, 159.

34. American Society for Testing and Materials (ASTM). Annual Book of ASTM Standarts, Standart Practices for Infrared Multivariate Quantitative Analysis - E1655-05, ASTM International, West Conshohocken, Pennsylvania, USA, 2012. 
35. Williams, P.; Em Near-infrared technology in the agricultural and food industries, $2^{\text {nd }}$ ed., Williams, P.; Norris, K.; Eds., American Association of Cereal Chemists Inc.: St. Paul, 2001

36. Faber, N. M.; Song, X. H.; Hopke, P. K.; TrAC Trends Anal. Chem. 2003, 22,330 .

37. Faber, K.; Kowalski, B. R.; Chemometr. Intell. Lab. Syst. 1996, 34, 283.

38. Zhang, L.; Garcia-Munoz, S.; Chemometr. Intell. Lab. Syst. 2009, 97, 152.

39. Pomeranz, Y.; Meloan, C. E.; Food Analysis: Theory and Practice, $3^{\text {rd }}$ ed., Chapman \& Hall: New York, 1994.
40. Workman Jr., J. J.; Weyer, L.; Practical Guide to Interpretative NearInfrared Spectroscopy, CRC Press: Boca Raton, 2008.

41. Kennard, R. W.; Stone, L. A.; Technometrics 1969, 11, 137.

42. Rinnan, A.; van den Berg, F.; Engelsen, S. B.; TrAC - Trends Anal. Chem. 2009, 28, 1201.

43. Horwitz, W.; Pure Appl. Chem. 1995, 67, 331.

44. Shahbazikhah, P.; Kalivas, J. H.; Chemometr. Intell. Lab. Syst. 2013 , 120,142 . 